\title{
Germanica
}

\section{Déformations typologiques et stratégies narratives}

Les contes et anticontes de Hans Carl Artmann

Typologische Verzerrungen. Erzählstrukturen in den Märchen und Antimärchen Hans Carl Artmanns

Jacques Lajarrige

\section{OpenEdition}

\section{Journals}

Édition électronique

URL : http://journals.openedition.org/germanica/1337

DOI : 10.4000/germanica.1337

ISSN : 2107-0784

Éditeur

Université de Lille

\section{Édition imprimée}

Date de publication : 1 janvier 1992

Pagination : 43-58

ISSN : 0984-2632

\section{Référence électronique}

Jacques Lajarrige, « Déformations typologiques et stratégies narratives », Germanica [En ligne], 11 |

1992, mis en ligne le 07 février 2014, consulté le 06 octobre 2020. URL : http://

journals.openedition.org/germanica/1337 ; DOI : https://doi.org/10.4000/germanica.1337

Ce document a été généré automatiquement le 6 octobre 2020.

(c) Tous droits réservés 


\title{
Déformations typologiques et stratégies narratives
}

\author{
Les contes et anticontes de Hans Carl Artmann \\ Typologische Verzerrungen. Erzählstrukturen in den Märchen und Antimärchen \\ Hans Carl Artmanns
}

Jacques Lajarrige

\author{
Es gibt einen Omnibus, \\ der fährt täglich \\ von Hansel nach Gretel. \\ Es gibt einen Schaffner, \\ der jede Hexe gratis mitfahren läßt. \\ Nach Feierabend \\ macht er halbe-halbe \\ mit den Gebrüdern Grimm. \\ (Günter Bruno Fuchs, Behauptungen ${ }^{1}$ )
}

I

1 L'association du nom du poète autrichien Hans Cari Artmann et du conte ne va pas sans paradoxe. Le genre exige en effet un récit ramassé, il se caractérise par la clôture qui en est l'une des constantes les plus universelles, et à laquelle l'écriture prolifique et souvent désordonnée de H.-C. Artmann paraît pour le moins inadaptée. Les textes artmanniens sont à l'inverse volontairement ouverts, toute notion d'achèvement leur est foncièrement étrangère, tant il est vrai que l'auteur cherche par divers moyens à laisser la porte ouverte à plusieurs significations du texte littéraire et à associer le lecteur à l'élaboration du sens. Pourtant, malgré ces réserves sur lesquelles il nous faudra revenir lorsque nous envisagerons des exemples précis, il faut bien constater que notre auteur, en connaisseur et lecteur averti, s'est intéressé de très près à la «mécanique » subtile du conte, comme en témoignent les textes auxquels il a lui-même attribué cette étiquette et la présence à tous les niveaux de l'œuvre de motifs et de 
personnages empruntés à ce genre. Si la magie du conte a exercé sur Artmann un pouvoir durable, c'est sans doute aussi parce qu'il est le carrefour idéal des grands mythes collectifs et des fantasmes privés, des structures sociales comme des visions personnelles, fortement marquées par le vécu conscient ou inconscient. A sa façon, Artmann fait apparaître le statut du conte dans toutes ses ambiguïtés, mais un conte est d'abord et avant tout un récit, un art du récit, et nous devrons nous intéresser de près aux stratégies narratives mises en œuvre par le poète et au statut que le récit artmannien attribue aux personnages des contes pour en déterminer la nature et les intentions. Pour cela, il nous faudra également interroger d'autres écrits du même auteur où l'emploi du mot conte n'apparaît pas comme intention d'écriture, mais qui seraient susceptibles d'éclairer la ou les significations que lui donne Artmann.

\section{II}

2 Il serait fastidieux de se livrer ici à un inventaire exhaustif des éléments thématiques et structurels issus des contes qu'offre une œuvre aussi éclectique que celle d'Artmann. Notons tout de même au passage que ses deux premières œuvres publiées ${ }^{2}$ témoignent déjà de façon abondante d'une influence marquée. Les épisodes du hussard dans Von denen Husaren und anderen Seil-Tänzern (GdR, I) regorgent de tailleurs, d'aubergistes, et autres rémouleurs tout droit sortis de l'univers merveilleux des contes, au même titre que les diablotins et sorcières qui, sous diverses apparences animales, proposent au hussard le redoutable pacte avec le Malin. Mais le hussard ne manque pas une occasion de clamer à qui veut l'entendre qu'il ne croit pas le moins du monde aux sorcières. Cependant, Von denen Husaren s'inscrit avant tout dans la tradition baroque européenne ${ }^{3}$ et doit beaucoup aux Schwänke. Ce qui nous intéresse ici particulièrement pour notre propos, c'est que ces histoires doivent être rangées dans la catégorie que Max Lüthi appelle «Märchenschwank» et dans laquelle il voit une tendance à la parodie, à la satire, à la défiguration du genre ${ }^{4}$. Les titres ultérieurs ne démentiront plus cette approche du conte, que ce soient les récits de rêves de Grünverschlossene Botschaft dominés par d'incessantes métamorphoses et enrichis par les conquêtes techniques $\mathrm{du} \mathrm{XX}^{\mathrm{e}}$ siècle, ou encore les comptines du cycle fantastique allerleirausch (ELB, 447-461) dont le titre indique à lui seul une approche humoristique des formulettes rimées qui ornent tant de contes.

On serait toutefois mal avisé de ne voir dans ces quelques exemples qu'un jeu gratuit ou un ornement facile du style de notre auteur. La lecture du récit en prose Nachrichten aus Nord und Sud (GdR, III) suffit au demeurant à montrer au contraire qu'Artmann ne prend pas les contes pour argent comptant et qu'il est aussi capable de les confronter à une analyse critique de leur réception, voire d'opposer le merveilleux qu'ils véhiculent à son expérience du monde. La vision du Rhin pollué lui fait dire par exemple :

à Düsseldorf, il me paraissait être une soupe gris-rat en provenance de la cuisine crasseuse de Cendrillon.

(GdR, III, 355)

tandis que la constatation pessimiste de l'âge qui approche à grands pas brise tout net la vision idyllique que l'on retient souvent de Blanche-Neige :

cette histoire de la jeunesse dorée est un artifice creux des adultes uhu colle tout c'est bien connu et le temps rouvre les vieilles blessures les sept nains eurent aussi une enfance plus qu'emmerdante l'épisode de Blanche-Neige n'est venu que beaucoup plus tard à l'âge mûr et finalement ça n'a pas dû non plus être un 
supertrip.

(GdR, III, 348)

En dépit de ces réserves au ton inhabituellement sévère pour Artmann qui va même jusqu'à mettre en cause la légitimité des contes à l'heure où il écrit ces lignes (GdR, III, 418), Nachrichten aus Nord und Süd témoigne encore et jusque dans la musique et le rythme des phrases ou formulettes - «alles freut sich wie gretel und zigretl im bettl»d'un envoûtement pour ce genre dont l'auteur ne voudrait pas priver sa fille Griseldis à qui il a acheté une version illustrée du recueil de Grimm.

\section{III}

Toutefois, Artmann ne s'est pas contenté de livrer des commentaires critiques sur la valeur des contes de son enfance, il en a également soumis quelques-uns à son incomparable talent parodique. Trois personnages de contes, la Barbe-Bleue, ToutesFourrures et Dame Holle, sont le point de départ de créations originales que H.C. Artmann n'a pas voulu désigner comme contes. Deux poèmes du recueil en dialecte viennois med ana schwoazzn dintn reprennent le thème de la Barbe-Bleue, le mari féroce et sanguinaire du conte de Perrault. Dès l'abord, un nombre important de différences sépare les deux héros homonymes. Chez Perrault, un roturier enrichi, chez Artmann, un propriétaire de manège pour enfants. Alors que le conte de Perrault ne dit rien du passé du personnage, si ce n'est qu'il a déjà épousé plusieurs femmes et "qu'on ne savait pas ce qu'elles étaient devenues ", le poème artmannien débute par l'aveu, de la bouche même du monstre qui parle à la première personne, de sept crimes commis pour se venger de sa première femme: elle l'a abandonné. S'ensuit une description circonstanciée du devenir des malheureuses épouses dépecées à la hache et enterrées sous le plancher de la chambre.

Le secret qui était chez Perrault l'un des ressorts de la narration est ici réduit à néant dès la première strophe de Blauboad 1. De même, le motif central de la chambre interdite où la Barbe-Bleue entasse ses victimes et dont il confie la clef ${ }^{5}$ à sa dernière épouse pour mettre sa curiosité à l'épreuve n'a plus ici de raison d'être. Pour cette même raison, la valeur exemplaire de la punition infligée par le mari dupé ne peut plus être la même. Les épouses du conte français paient successivement pour un délit que chacune d'elles commet tour à tour alors que le poème fait porter la punition qui revient à la première par les suivantes bien qu'elles n'aient d'autre responsabilité que d'avoir accepté l'invitation à "un rêve d'amour ». Mais ici, le bourreau des cœurs ne s'arrête pas au nombre fatidique, si souvent rencontré dans les contes, de sept victimes. Une huitième, puis sans doute d'autres encore, devra subir le supplice du tranchoir sans pouvoir compter sur l'aide bienveillante d'une sœur Anne ou de deux frères.

Son assurance arrogante se voit vite tournée en dérision lorsqu'à la dernière strophe, il avoue avoir peur du noir et ne pouvoir dormir sans lumière par crainte de ses victimes. Le plus intéressant réside ici pour nous dans l'inversion apparente de la morale qui punit le coupable criminel et non la coupable victime de curiosité. Mais cette issue ne peut faire qu'illusion, car en réalité, la peur exprimée ne suffit pas à suggérer le remords, tout au plus peut-elle ridiculiser le « héros ». Sans perdre de vue le fait que la poésie lyrique opère forcément avec d'autres moyens qu'un récit du type du conte, il faut souligner qu'Artmann ôte au modèle auquel il a recours sa progression dramatique en livrant l'horreur comme une banalité qui semble aller de soi. Il nous propose dans ce 
poème un étonnant mélange de terreur et de ridicule dont la conséquence est que le lecteur ne peut plus considérer le personnage de la Barbe-Bleue dans la seule optique du merveilleux. Le dialecte viennois dont la réalisation phonétique a quelque chose d'inquiétant favorise d'ailleurs et amplifie ce télescopage de registres antinomiques jusqu'à faire glisser le motif d'emprunt sur la pente d'une virulente satire des Viennois, comme le confirment d'autres poèmes de ce recueil par ailleurs étrangers à l'univers des contes. Le conte est donc bien mis ici au service d'un projet d'écriture plus large qui n'a plus grand-chose à voir avec l'origine dont il se réclame pourtant ouvertement. Le poème Blauboad 2, une mise en scène à la première personne d'une boucherie sanguinaire, ne se différencie pas foncièrement du premier. Là encore sont contés les rêveries éveillées d'un assassin dont on ne saurait dire au juste s'il est véritable ou seulement potentiel. Artmann franchit avec ce deuxième exemple un pas supplémentaire dans l'inversion de la critique morale du conte en critique sociale au détriment de sa ville natale et de ses concitoyens :

dans une société comme celle-là on ne peut me faire confiance je suis en proie à un

cafard noir comme du charbon !...

(Msd, 18)

7 Contrairement au mari châtié du conte de Perrault ou à celui de Fitchers Vogel, le meurtrier savoure à l'avance la médiatisation de ses forfaits et affiche l'assurance effrontée de pouvoir réintégrer le cadre de la vie sociale sans être inquiété. On voit bien à quel point le « moi lyrique » joue avec toutes les ambiguïtés offertes par le modèle, et on comprend mieux, dès lors, qu'il est principalement un être de papier au service de l'auteur dont la fonction avant tout littéraire est de jeter le trouble, de créer la distance par rapport à la scène du crime dénaturé et en même temps d'abolir symboliquement cette distance par le seul fait de sa participation directe à l'intrigue en tant que personnage principal et unique qui ne laisse à aucun moment la parole aux femmes. Ce n'était pas le cas chez Perrault.

8 C'est une approche sensiblement différente qu'adopte le poète en proposant dans le soixante-dix-septième rêve de Grünverschlossene Botschaft les aventures de ToutesFourrures. En effet, la réécriture du conte de Grimm Allerleihrauh ne porte plus tant sur la personnalité de la jeune fille du conte que sur la nature du récit qui conserve de l'original les seuls motifs ou détails propres à faire ressortir les intentions profondes de H.-C. Artmann. Outre la parenté de nom entre les protagonistes, le refuge de l'arbre creux, à la fois symbole de féminité et de puissance virile, le récit de rêve conserve, avec d'importantes modifications, l'épisode des chiens qui découvrent la cachette de Toutes-Fourrures. Mais la parenté s'arrête à peu près là. Artmann occulte volontairement la mort de la mère, les circonstances donc, qui ont conduit la jeune fille à fuir la demande en mariage d'un père incestueux et prive ainsi le conte d'un aspect essentiel qui en permettait la dynamique narrative et en constituait toute la signification. Ainsi, c'est la trajectoire symbolique d'une adolescente accédant à l'âge adulte et découvrant la sexualité qu'une mise en scène à peine voilée de l'auteur en séducteur incorrigible (A \& C. lire $\mathrm{H}[\mathrm{ans}] \mathrm{C}[\mathrm{arl}])$ réduit à son plus strict minimum, à savoir la cruauté de l'acte sexuel imposé par la force. Ajoutons à cela que l'auteurnarrateur se substitue (en rêve) au père-chasseur qui retrouve sa fille par hasard, et invalide du même coup le soupçon d'inceste. Le message délivré est loin d'être univoque : en réduisant les préliminaires du conte à néant, H.-C. Artmann permet au lecteur d'accéder de plain-pied à une compréhension immédiate des mécanismes symboliques du rêve-conte et de ses contenus latents, mais cette méthode est bien sûr 
redevable pour une large part à la psychanalyse dont l'auteur démasque par la même occasion la rigidité interprétative obnubilée par la notion d'€dipe. Corrélativement, ce que Pierre Péju a appelé la "puissante moulinette psychanalytique ${ }^{6}$ a également broyé les accessoires merveilleux du conte (anneau d'or, petit rouet d'or, dévidoir d'or, les trois robes, la soupe cuisinée pour le père-roi etc.). Toutes ces soustractions permettent en définitive, à l'instar du rêve qui ne procède pas autrement, de condenser le récit, de le ramener à ce qu'au fond il suggère par le biais de multiples méandres. En contrepartie, l'espace ainsi libéré est investi par des ajouts qui dénotent de stratégies narratives nouvelles, contraires à celle du conte. Le récit de rêve donne un caractère véridique au texte qui se traduit à l'intérieur de ce que nous pourrions presque appeler un métaconte par des précisions de date ou une localisation géographique - le rêve doit impérativement se dérouler en Russie, en 1877 - même si par ailleurs les coordonnées spatio-temporelles n'ont aucun rapport nécessaire avec l'action qui pourrait tout aussi bien se dérouler ailleurs et en d'autres temps. Malgré ces efforts appuyés pour arracher le conte à l'atemporalité du "il était une fois », la tentative d'adéquation entre le réel et le rêve hypothétique chargé de désirs libidinaux, tourne rapidement en faveur de ce dernier. Les différences avec le conte traditionnel sont assez évidentes. Le locuteurnarrateur des contes s'affirme généralement comme le porteur d'un récit hérité de la tradition orale qu'il ne fait que transmettre, plus que comme l'inventeur de ce récit. A l'inverse, le rêve artmannien fait apparaître l'écrivain comme le héros conteur, suggérant par là que la fiction narrative tire sa cohérence de ce que l'aventure a été transmise par l'activité onirique. En donnant à plusieurs reprises à lire son origine, elle se donne d'une certaine façon pour véridique. La formule « en rêve " qui accompagne chaque verbe d'action, permet de réactualiser le récit à volonté au même titre que le « il était une fois... ». Enfin, et ce n'est pas l'aspect le moins significatif, le récit de rêve se veut délibérément ouvert comme les dernières lignes le suggèrent très bien :

Et tu la violentes (en rêve) et le grand-duc entend ses soupirs en rêve et il se réveille (en rêve) [...] mais tu es à soixante-dix-sept verstes de là, et ce nombre est aussi le numéro qui confère quelque signification à ce rêve, bien que tu doives calculer par toi-même le comment et le quand?.

Comme souvent avec Artmann, ce genre d'appel au lecteur doit être pris avec toute la circonspection et l'humour qui s'imposent car la parodie tourne une nouvelle fois à l'autoparodie. Ce que le lecteur est censé décider de son propre chef figure déjà dans le récit, la signification du rêve est traduite en toutes lettres. Reste à prendre acte de la distance qui nous sépare du conte: 77 !, c'est-à-dire, $7+7$, ou deux fois le conte Allerleihrauh.

10 Le récit fantastique Frankenstein in Sussex débute lui aussi par une mise en garde au lecteur dans lequel l'auteur précise que les événements qui vont suivre n'ont rien d'une invention et qu'ils n'ont pas été davantage empruntés à une fiction déjà existante (GdR, II, 393). De quoi s'agit-il au juste? De la petite Alice qui n'est autre que celle de L. Carroll : par curiosité, elle regarde par l'ouverture d'une cheminée, tombe dans les entrailles de la terre et atterrit dans un chaudron rempli de soupe aux cygnes. Alice se trouve dans la maison d'un ogre, monstre artificiel ou golem, qui veut s'en prendre à sa pureté et la soumet à diverses épreuves. Celui-ci avoue être le fils du Docteur Victor von Frankenstein. Pendant ce temps, Mary Wollstonecraft Shelley, la mère spirituelle du monstre en question ${ }^{8}$ lit un extrait de conte à Dame Holle confortablement installée dans un salon du firmament. Il s'agit en réalité d'un collage de citations tirées du conte de Grimm Les six cygnes. Quelques lignes suffisent à ennuyer Dame Holle qui, signe des 
temps, préfère regarder la télévision pour s'informer de la marche du monde. C'est par cet intermédiaire que les deux femmes suivront la chasse à la jeune vierge auquel se livre l'ogre et les tentatives du gentleman John Hamilton Bancroft pour sauver Alice des griffes de celui-ci. A ce niveau déjà, deux constatations s'imposent : 1) Dame Holle a déserté le conte auquel elle doit son existence, 2) elle préfère ne pas entendre un conte frelaté. Mais il est vrai qu'Artmann prend soin de préciser que :

dame Holle n'est pas, comme on le raconte à tort dans les histoires pour enfants, une vieille bonne femme mais plutôt une femme d'une beauté envoûtante aux yeux bleus et aux cheveux fournis, blonds comme les blés, coupés et coiffés chaque fois à la dernière mode !

(GdR, II, 395-396)

Cette différence se confirme en partie lorsque Dame Holle, qui suit l'évolution du conflit entre l'ogre et Alice devant son récepteur de télévision, s'oppose fermement à ce que le monstre s'empare de la petite Alice, empêchant ainsi que la loi inexorable des contes et des chasses fantastiques ne s'accomplisse. Dame Holle serait-elle par hasard une empêcheuse de conter en rond? Du conte qui porte son nom, elle a toutefois conservé les pouvoirs surnaturels d'une bonne fée. Son indignation initiale cède bientôt la place à la décision ferme d'intervenir en personne (GdR, II, 405). Par glissements progressifs, elle quitte donc au fil du récit son rôle de simple spectatrice pour s'impliquer de plus en plus directement dans l'évolution du conflit. D'abord en usant de ses pouvoirs magiques dont elle fait bénéficier Alice ou Bancroft : comme aux six frères du conte Les six cygnes, elle fait pousser des ailes à Bancroft, doublure ridiculisée de l'auteur-séducteur, puis voyant que cet artifice ne suffit plus, elle intègre la fiction artmannienne comme personnage (actant) à part entière. Pour empêcher que le dessein de la romancière fantastique $\mathrm{M}$. Shelley ne se réalise, Dame Holle décide de l'enfermer dans la salle de bains, de prendre les commandes d'un sous-marin pour enlever Bancroft et Alice. Le monstre de Shelley qui attend une fiancée depuis cent cinquante ans (50 de plus que le prince de la Belle au Bois Dormant!) n'aura à la fin d'autre solution que de "dévorer » sa mère spirituelle. Ce singulier combat réunit en fait par personnages littéraires et écrivains interposés, deux tendances emblématiques de l'écriture artmannienne. Littérature fantastique versus conte de fées? Les choses ne sont pas aussi tranchées bien sûr, car si Mary Shelley succombe à son héros, ce n'est pas seulement parce que le fantastique, comme le conte du reste, peut être autophage, se nourrir à l'infini de ses représetations mentales et de ses structures formelles. L'issue ne peut davantage être interprétée comme l'expression d'une préférence d'ordre littéraire ou esthétique de H.-C. Artmann pour le conte, car à bien y regarder, la logique fantastique se trouve malgré tout réalisée par des voies détournées et ironiques aux dépens du conte pur.

11 Si nous tentons de dresser un bilan provisoire, nous constatons que Dame Holle est ici tour à tour héros de conte déchu de son statut de personnage, spectateur extérieur d'une fiction qui n'est nullement un conte pour finalement redevenir l'acteur principal d'un dénouement « heureux ». Mais les différents commentaires auto-ironiques dont le narrateur assortit son récit montrent bien que Dame Holle n'est plus rien qu'un instrument; en d'autres termes et pour employer la terminologie de Propp, l'actant " Dame Holle » est ici réduit à une « fonction narrative », un moteur qui participe d'une dynamique fictionnelle plus complexe et plus éloignée du conte. Ainsi revu et corrigé, l'héritage du conte ouvre la voie à de nouvelles virtualités narratologiques et contribue, 
c'est bien le sens profond du combat symbolique Shelley/Holle, à l'élaboration de l'écriture fantastique.

De l'étude de ces trois exemples, il ressort que l'attitude réceptive et créatrice de H.C. Artmann vis-à-vis des contes est loin d'être univoque et simpliste. Le poète semble expérimenter les diverses possibilités littéraires qu'offre ce genre pour son œuvre. Il tente tout à la fois de créer et d'abolir la distance qui le sépare des motifs et des personnages du conte. Il l'abolit parfois en faisant jouer aux personnages tout ou partie de leurs rôles de personnages de conte, comme nous l'avons constaté pour Blauboad ou Allerleirauh, en n'épuisant pas les thèmes narratifs auxquels il se réfère. Ce procédé s'accompagne d'un corollaire: Artmann se donne comme auteur-narrateur un rôle de personnage ou suggère une identité qui laisse planer le doute de l'identification toujours possible. Les noms de personnages, les motifs connus, revêtent une fonction d'« enclencheurs " dans une structure narrative dont les caractéristiques essentielles sont dénaturées. Ces éléments créent chez le lecteur un effet de familiarité qui permet de préparer le terrain d'une «Verfremdung» qui n'en sera que plus efficace. A côté de cela, Artmann crée une distance par divers artifices comme le ton parodique qu'il adopte, la mise en relief ironique de l'écrivain séducteur. L'usage contradictoire des astuces du conteur ressort naturellement avec plus de force encore, lorsqu'il feint de prendre l'exact contre-pied de la manière traditionnelle, car c'est alors pour mieux en mettre en lumière le fonctionnement à ses yeux sans doute trop figé et répétitif. On peut donc affirmer que la réécriture du conte s'interroge aussi sur le rapport avec la réalité sociale des constellations stéréotypées qu'elle met en jeu. Les relations facétieuses de H.-C. Artmann à l'univers des contes, les mystifications auxquelles il les soumet, ironisent sur leur prétention à moraliser, à canaliser les pulsions et les désirs, indiquant par avance que la nécessité de la morale salutaire et éducatrice, cette "machine à socialiser", doivent être pris avec ironie et grande liberté. D'où les moralités en trompe-l'œil proposées dans Frankenstein in Sussex et les poèmes de la Barbe-Bleue. Peut-être au fond, et ce n'est pas là la moindre surprise qu'offre la confrontation à laquelle nous avons soumis les textes artmanniens, notre auteur retrouve-t-il ou recrée-t-il, à son insu sans doute, certaines conditions de l'or alité qui, nous le savons bien, est l'origine même des contes, en cela qu'il attire le lecteur sur de fausses pistes, qu'il lui offre de prendre une part active dans l'élaboration narrative du conte, tout en l'empêchant de s'identifier au(x) personnage(s). Les dissonances sont autant d'invitations à la méfiance et au doute. En prenant parfois les contes au pied de la lettre, il les fait échapper à une fixité qu'ils n'ont acquise que tardivement par l'écriture et la transmission littéraire.

\section{IV}

13 Les récits auxquels Artmann a lui-même donné le nom de conte doivent également, nous semble-t-il, être envisagés sous ce même angle du rapport à la réalité sociale qu'ils induisent. Le Märchen vomprinzen (GdR, I, 20) nous transporte d'emblée dans la féerie des contes: un prince se promène à cheval lorsqu'un condor laisse échapper deux souliers d'argent. Malgré l'intervention du renard, lequel lui conseille vivement de mettre pied à terre pour les ramasser, le fier cavalier poursuit sa chevauchée. Peu après, il rencontre sur le bord d'un chemin une sorcière qui s'est brisée la jambe et miaule comme un chat. Le prince fait alors montre de bienveillance et lui confectionne 
une attelle. Pour tout remerciement, la sorcière le change en caillou. Nous retrouvons ici concentrés tous les éléments constitutifs du conte: un prince charmant, le merveilleux sous diverses manifestations (souliers d'argent, métamorphoses...) une sorcière jeteuse de sorts, des animaux personnifiés, des personnages en situation de conflit etc. Dans leur ensemble, ces accessoires sont destinés à voiler le sens premier du conte. D'autres caractéristiques formelles sont instructives. Le conte du prince renonce en effet à se donner pour tel dès le début. Artmann prend le lecteur de vitesse et le transporte directement au cœur de l'action. Mais là encore, le plus important reste l'issue du récit. Celui-ci s'achève sur une inversion de la morale qui veut que tout se termine pour le mieux dans le meilleur des mondes et que, d'autre part, le Bien soit récompensé, le Mal châtié. Le prince qui refuse d'interrompre sa course pour prendre les souliers d'argent ne répond pas à l'appel, pourtant univoque, du merveilleux et prive le conte d'une happy-end. Il semble peu logique cependant qu'il en soit puni par la sorcière à qui il a porté secours. Le texte délivre un message crypté. La sorcière pourrait bien être une bonne fée, peut-être celle-là même qui aurait pu chausser les souliers d'argent, ce qui lui aurait sans aucun doute évité de se briser les os. Elle n'a dans l'économie générale du conte d'autre fonction que d'enseigner au mauvais prince à se défaire des œillères qui le confinent dans l'étroitesse du quotidien et de l'amener sur la voie du rêve et du surnaturel.

En le figeant en pierre, la vieille ne fait que matérialiser et extérioriser la vraie nature de cet esprit obtus. Autrement dit, sans la volonté de dépasser les catégories logiques dictées par la raison, le voyage (ici la chevauchée prise comme emblème de pulsion sexuelle) n'a plus de sens, il se condamne de lui-même à l'immobilité. Malgré les apparences, ce faux conte en est un vrai puisqu'il conserve toute l'authenticité de ses fonctions pédagogiques, et dans le cas présent prophylactiques. Toute l'originalité de l'écriture artmannienne réside dans l'inversion des schèmes archétypes (mauvais prince, bonne sorcière) pour finalement en conserver une fonction moraliste 9 .

Si nous avons choisi de conclure cette étude du conte chez Artmann par le cycle H.C. Artmannsens Märchen (GdR, I, 335-337), c'est parce qu'il propose une approche et une écriture des contes radicalement opposées à ce que nous avons déjà rencontré. On reconnaîtra dans le titre une référence limpide à Andersen à travers laquelle Artmann témoigne ici de son admiration pour le conteur danois ${ }^{10}$. Chacun de ces mini-contes a bien sûr sa spécificité, mais nous nous efforcerons ici seulement de ne retenir que quelques constantes communes qui nous paraissent de nature à éclairer un projet d'écriture. Ces textes ne quittent jamais totalement le domaine traditionnel du conte, et pourtant, ils s'en distinguent par un nombre significatif de points qui tiennent essentiellement à leur mise en texte. Ces récits brefs accèdent sous la plume d'Artmann à une simplicité de forme qui, fait rare chez cet auteur plus familier de la surcharge baroque que de la formulation limpide et lapidaire, ressemble à une "épuration » du conte qui se traduit avant tout par des signes de narrativité et des procédés stylistiques précis : sobriété de l'expression et de la fiction, lorsqu'elle existe, refus des transitions et des détails susceptibles de faire progresser le récit... Comme le suggèrent les énumérations dans Samson der treue maat von der Sancta Habana et Robinson Crusoes materialien, qui répertorient respectivement les outils nécessaires à la construction d'un moteur de bateau et les ustensiles du navigateur échoué, le conte artmannien est un jeu de construction comme un autre. On pourra objecter que les contes traditionnels ne sont peut-être rien d'autre, mais même si l'action y est resserrée, les personnages 
définis et qualifiés de façon sommaire, ils développent au moins une ligne narrative unique qu'ils mènent à un dénouement en passant par toutes les étapes nécessaires à leur compréhension. C'est à tout cela à la fois que les contes de H.-C. Artmannsen renoncent, avec pour conséquence l'abandon du merveilleux, de l'enchantement ambigu provoqué par la peur, de la mise en énigme de fantasmes et de rêveries. Même lorsqu'à l'instar du récit Ein schöner märztag, le lecteur se croit enfin en présence d'une situation reconnue comme pouvant appartenir à un conte - un jeune homme à qui un malheur vient d'arriver se met en chemin et tombe sur une sorcière - il doit se résigner à voir l'histoire tourner court :

Il souhaita le bonjour à la petite vieille et lui demanda comment ça allait.

Oh, lui répondit l'horrible sorcière, joli temps de mars et pas un cent dans mon

cochon-tirelire...

(GdR, I, 336-337)

La sorcière, vestige affaibli et pâlot de l'univers des contes n'a en effet plus d'autre pouvoir que de constater l'état du temps et de sa maigre fortune. Et le narrateur de s'apitoyer ironiquement sur le pauvre homme qui avait l'intention de tuer la sorcière et se voit réduit à démonter son pistolet pièce pour le jeter dans une lagune puante. En peu de lignes, Artmann fournit une recette pour rater sans coup férir l'écriture d'un conte et réussir celle d'un anticonte.

\section{V}

"Cric, Crac, mon conte est achevé... », annonce parfois le conteur. Notre promenade à la lisière des contes et anticontes artmanniens s'achève elle aussi, sur ce constat d'évidence : la manière de conter lui importe souvent plus que ce qu'il conte, même s'il est vrai qu'il ne peut être pour lui question de reproduire servilement des schémas narratifs, pas plus d'ailleurs que les contenus qu'ils induisent. L'écriture du conte, sa réécriture parfois poussée jusqu'à la négation, s'affirment comme le lieu privilégié d'échanges diégétiques interactifs capables de faire entrer des éléments connus en combinaison avec des inventions loufoques au sein de configurations nouvelles, d'explorer les ressources des jeux de rôles et leurs implications sociales. Si comme l'écrit Claude Brémond le " génie du conte est dans cette aptitude au délire contrôlé " ${ }^{11}$, reconnaissons que le délire d'Artmann est celui d'un conteur de génie qui, comme le contrôleur d'omnibus du poème de G.-B. Fuchs, sait rendre aux frères Grimm la part qui leur revient.

\section{NOTES}

1. Les abréviations des œuvres de H.-C.Artmann renvoient aux éditions suivantes: GdR : Grammatik der Rosen, Gesammelte Prosa, 3 Bände, Residenz Verlag, Salzburg, Wien, 1979.

ELB : Ein lilienweißer brief aus lincolnshire, gedichte aus 21 jähren, Suhrkamp Taschenbuch 498, Frankfurt/M, 1969.

Msd: Med ana schwoazzn dintn, Otto Müller Verlag, Salzburg, 1958. 
- Günter Bruno Fuchs, Behauptungen, in Gemütlich summt das Vaterland, Carl Hanser Verlag, München, Wien, 1984, p. 10.

2. - Med ana schwoazzn dintn, Otto Müller Verlag, Salzburg, 1958 et Von denen Husaren und anderen Seil-Tänzern, Piper, München, 1959.

3. - A ce sujet, nous renvoyons à notre étude : H.-C. Artmann, tradition littéraire et exercices de style. La mémoire ouverte ou la mort déjouée. Introduction à une lecture de l'œuvre. Thèse de Doctorat, Lille III, 1988.

4. - Max Lüthi, Märchen, Sammlung Metzler 16, Metzlersche Verlagsbuchhandlung, Stuttgart, 1962, notamment le chapitre Abgrenzung gegen benachbarte Gattungen: Sage, Legende, Mythus, Fabel, Schwank, p. 7 sq. Lüthi voit dans le Schwank non pas un genre en soi mais la possibilité d'une variante d'autres genres (Legendenschwank, Sagenschwank...). De nombreux épisodes de Von denen Husaren correspondent à la définition du Märchenschwank qui selon Lüthi modifie l'image du monde présentée par le conte et remplace les miracles par les astuces du héros.

5. - Dans la version allemande du Fitchers Vogel du recueil des frères Grimm, le pendant du conte de Perrault, c'est un œuf et non une clef-fée, qui conserve les traces de sang et trahit l'épouse curieuse.

6. - Pierre Péju, La petite fille dans la forêt des contes, Robert Laffont, Paris, 1981, p. 60.

7. - Nous citons d'après notre traduction: H.-C.Artmann, Message viride, 90 rêves. Editions Jacqueline Chambon, Nîmes, 1991.

8. - Mary W. Shelley est l'auteur du roman d'horreur Frankenstein ou Un Prométhée moderne (1819) cité par Artmann, et donc la mère spirituelle du personnage qu'il réutilise dans sa propre fiction.

9. - La morale n'est pas ici fidélité à une forme choisie, mais refus d'un comportement social policé, uniquement guidé par la raison.

10. - Artmann nous a précisé que c'est par référence à Hans Christian Andersen qu'il a opté pour l'orthographe de son prénom avec un C (Hans Carl), ce qui donne les mêmes initiales pour les deux écrivains : H.C.A. Les récits du cycle H.-C. Artmannsens Märchen ont été écrits pour un livre de contes qui n'a jamais vu le jour et qu'Artmann aimerait aujourd'hui encore achever. Propos recueillis dans un entretien avec l'auteur à Vienne, le 23.8.1991.

11. - Claude Brémond, Le Meccano du conte, in Magazine littéraire, $n^{\circ} 15$, juillet-août 1979, p. 16.

\section{RÉSUMÉS}

La recherche de la présence du conte dans la production abondante de H.C. Artmann laisse apparaître que le jeu des références et des emprunts tant thématiques que structurels par lequel elle se manifeste dès les premières publications n'est nullement synonyme de reproduction fidèle ou de réception non-critique de modèles canoniques. Artmann va même jusqu'à s'interroger dans Nachrichten aus Nord und Süd sur la légitimité des contes de son enfance qu'une confrontation au monde d'aujourd'hui rend problématique.

Par comparaison avec les « contes-modèles » dont ils sont les protagonistes, l'analyse du statut des personnages artmanniens de la Barbe-Bleue, de Toutes-Fourrures et de Dame Holle permet de dégager un projet de réécriture fantastique du conte qui accorde la priorité à la parodie et à l'humour, et de systématiser les attitudes de l'auteur vis-à-vis de l'univers des contes. L'originalité réside ici avant tout dans les modalités de renonciation ainsi que dans la distance critique que les stratégies narratives mises en œuvre et les transgressions incessantes des 
frontières du conte imposent au lecteur.

Les contes écrits par Hans Carl Artmann dénotent quant à eux deux positions fondamentales : celle d'abord qui consiste par l'inversion de la morale à refuser de voir dans le conte une «machine à socialiser", la seconde, plus radicale, qui pousse les déformations typologiques à l'extrême, au point d'aboutir à l'écriture d'anticontes.

Schon im Frühwerk Hans Carl Artmanns stößt der Leser auf Spuren des Märchenhaften; eine eingehendere Untersuchung seines umfangreichen Schaffens zeigt jedoch, daß es sich dabei weder um eine unkritische Rezeption noch um getreue Nachahmung festgeschriebener Formen und tradierter Modelle handelt. In Nachrichten aus Nord und Süd konfrontiert Artmann sogar das Idyllische der Märchen seiner Kindheit mit seiner eigenen Welt er fahrung.

In einer Gegenüberstellung der von H.C. Artmann entliehenen Figuren Blaubart, Allerleirauh und Frau Holle mit ihrem Original wird eine Technik der literarischen Um-Schreibung sichtbar, die der Parodie und der Selbstironie verpflichtet ist und aus der sich Rückschlüsse auf typische Einstellungen des Autors zum Märchen ableiten lassen.

Artmanns besondere Erzählstrategien, seine Gratwanderung entlang der Grenzen überlieferter Vorlagen verlangen vom Leser eine Revidierung seines Märchenkonzepts.

Auffällig sind in Artmanns Märchen zwei Formen der Stellungnahme: durch Umkehrung der Moral wird die erzieherische Rolle des Märchens als Soziali-sierungsinstanz in Frage gestellt, bzw. ad absurdum geführt, extreme typologische Verzerrungen können sogar zur Entstehung von Antimärchen führen.

\section{AUTEUR}

\section{JACQUES LAJARRIGE}

Université Blaise Pascal, Clermont II 